\title{
PROFIL VON PATIENTEN, DIE IN EINER AMBULANZ FÜR MEDIZINISCHE GENETIK IM UNIVERSITÄTSZENTRUM VON BELÉM, PARÁ, AMAZONIEN, BEHANDELT WURDEN
}

\section{ORIGINALER ARTIKEL}

LIMA, Gabriela Elenor dos Santos ${ }^{1}$, LOPES, Carlos Henrique Martins², DENDASCK, Carla Viana ${ }^{3}$, OLIVEIRA, Ciane Martins de ${ }^{4}$, OLIVEIRA, Euzébio de ${ }^{5}$

LIMA, Gabriela Elenor dos Santos. Et al. Profil von Patienten, die in einer Ambulanz für medizinische Genetik im Universitätszentrum von Belém, Pará, Amazonien, behandelt wurden. Revista Científica Multidisciplinar Núcleo do Conhecimento. Jahrgang 06, Ed. 03, Vol. 11, S. 48-62. März 2021. ISSN: 24480959, Zugangslink:

https://www.nucleodoconhecimento.com.br/gesundheit/medizinische-genetik, DOI: 10.32749/nucleodoconhecimento.com.br/gesundheit/medizinische-genetik

\section{ZUSAMMENFASSUNG}

Medical Genetics (GM) hat sich zu einer anerkannten medizinischen Spezialität entwickelt, mit wichtigen Konzepten und Ansätzen bei der Diagnose und Behandlung vieler häufiger und seltener Krankheiten. Genetische Krankheiten folgen Vererbungsmustern und können autosomal rezessiv, autosomal dominant, mit dem X-Chromosom oder Y-Chromosom verbunden oder multifaktoriell sein. Ziel dieser Studie war es, das Profil der Patienten zu ermitteln, die in einer GM-Ambulanz in einem Universitätszentrum in Belém (Bundesstaat Para) behandelt wurden. Die

\footnotetext{
${ }^{1}$ Medizinischer Akademiker am Universitätszentrum des Staates Pará - CESUPA.

${ }^{2}$ Medizinstudent am Universitätszentrum des Bundesstaates Pará - CESUPA.

${ }^{3}$ Theologe, PhD in Clinical Psychoanalysis. Er arbeitet seit 15 Jahren mit Scientific Methodology (Research Method) in der wissenschaftlichen Produktionsberatung von Master- und Doktoranden. Der Spezialist für Marktforschung und -forschung konzentrierte sich auf Gesundheit.

${ }^{4}$ PhD in Medizinischer Genetik. Professor und Forscher am Universitätszentrum des Staates Pará (CESUPA).

${ }^{5}$ PhD in Medizin/Tropische Krankheiten. Professor und Forscher an der Föderalen Universität Pará - UFPA.
}

RC: 79877

Disponível em: https://www.nucleodoconhecimento.com.br/gesundheit/medizinische- 
Daten wurden aus Patientenakten gesammelt, die zwischen 2014 und 2019 anhand des eigenen Fragebogens der Forscher beobachtet wurden, wobei die Daten über das Microsoft Excel-Programm analysiert und tabelliert wurden. Insgesamt wurden 101 Krankenakten analysiert, wobei Frauen (51 Patienten) vorherrschen. Darüber hinaus wurde der größte Teil der Betreuung für Kinder (41,5 \%) in Den Vordergrund gedran. Hinsichtlich der ethnischen Zugehörigkeit wurden nur die Variablen "weiß" und "braun" beobachtet, mit einer höheren Prävalenz von braunen Patienten (78 der Gesamtzahl). Auch Belém war die am weitesten verbreitete Stadt in der Natürlichkeit der Patienten (61 Datensätze). Die Spezialgebiete mit der höchsten Anzahl von Überweisungen an die GM -Ambulanz waren Endokrinologie und Neurologie, wobei neuropsychomotorische Entwicklungsverzögerung die häufigste Diagnose war. In 42 Krankenakten war das Alter bei der Diagnose nicht vorhanden. Von den 101 Patienten hatten nur 16 genetische Beratung und in den übrigen 85 gab es keine Aufzeichnungen über diese Informationen. Schließlich wurde das mütterliche Alter bei der Geburt in den meisten Krankenakten nicht gefunden (abwesend in 61,38\%). Daher ist es wichtig, ein Profil des Patienten zu entwickeln, das in einer GMAmbulanz gesehen wird, da es möglich wird, fehlerbefähigte Leistungen im Service zu identifizieren, zusätzlich zur Anpassung der Arzt-Patienten-Beziehung.

Schlagworte: Medizinische Genetik, Ambulanz, genetische Erkrankungen.

\section{EINLEITUNG}

Genetik entstand in der Medizin im frühen zwanzigsten Jahrhundert, als man erkannte, dass mendelianische Gesetze der Vererbe das Wiederauftreten einiger familiärer Störungen erklären könnten. In den nächsten 100 Jahren hat Medical Genetics (GM) aufgehört, eine kleine Unterspezialität zu sein, die sich nur auf wenige seltene Erbkrankheiten konzentriert, um eine anerkannte medizinische Spezialität zu werden, mit wichtigen Konzepten und Ansätzen bei der Diagnose und Behandlung vieler häufiger und seltener Krankheiten (NUSSBAUM et al., 2016).

RC: 79877

Disponível em: https://www.nucleodoconhecimento.com.br/gesundheit/medizinische- 
GM wurde 1983 vom Bundesrat als medizinische Spezialität anerkannt und 1986 wurde die Brasilianische Gesellschaft für Medizinische Genetik gegründet, um das Wissen über die Spezialität zu erweitern. In jüngerer Zeit hat GM in der genetischen Beratung relevant gehandelt (SOCIEDADE BRASILEIRA DE GENÉTICA MÉDICA, 2020).

Nach Angaben der Weltgesundheitsorganisation (WHO) betrifft die genetische Beratung eine genetische Klärung, die darauf abzielt, Genotypen, die für Krankheiten oder Geburtsfehler verantwortlich sind, durch prospektive oder retrospektive Identifizierung von Vereinigungen zu verhindern, die diese Veränderungen herbeiführen können. Darüber hinaus umfasst die Beratung auch die Meldung von Problemen im Zusammenhang mit dem Auftreten oder dem Risiko einer genetischen Erkrankung, die in der Familie auftritt. Daher ist es notwendig, in einem multidisziplinären Team von Fachleuten in der Lage, den Patienten und seine Familie in Bezug auf die Diagnose, den Verlauf der Krankheit, die verfügbaren Ansätze, das Risiko eines Wiederauftretens und Alternativen zu einem solchen Risiko zu beraten. In diesem Zusammenhang wird hervorgehoben, dass der Fachmann, der Beratung anbietet, den von ihm behandelten Patienten kein Verhalten vorschlagen, angeben oder verlangen kann (BERTOLLO et al., 2013).

Eine Krankheit genetischen Ursprungs geht auf Veränderungen in den Sequenzen von Deoxyribonukleinsäure (DNA), dem genetischen Material jedes Einzelnen oder Mutationen auf der Ebene sexueller oder autosomaler Chromosomen (GRIFFITHS; WESSLER; DOEBLEY, 2016).

Genetische Krankheiten folgen Vererbungsmustern, können sein: (1) autosomal rezessiv, die sich nur bei homozygoten Individuen für ein autosomales Gen manifestieren, d. h. betroffene Individuen haben zwei Kopien des mutierten Gens, wie Zwielichtige Fibrose, die aus einer Mutation im CFTR-Gen auf Chromosom 7 resultiert, und klassische Phenylketonurien, die mit Mutationen im Chromosom auf dem Chromosom 12 zusammenhängen; (2) autosomal dominant, bei dem mutierte

RC: 79877

Disponível em: https://www.nucleodoconhecimento.com.br/gesundheit/medizinischegenetik 
Gene auch auf Autosome-Chromosomen stehen, jedoch kann eine einzige Genkopie die Krankheit verursachen, wie die Huntington-Krankheit; (3) X-verknüpfte, auch als geschlechtsbezogene Vererbungen bekannte Chromosomen befinden sich in einem nicht-homologen Bereich des X-Chromosoms und Mutationen sind bei Männern dominant, die Hauptbeispiele sind Farbblindheit, Hämophilie und DuchenneMuskeldystrophie; (4) verbunden mit Y, oder hol-ndrica-Vererbung oder Vererbung, die auf das Geschlecht beschränkt ist, in dem das Auftreten der Krankheit mit dem YChromosom in seinem nicht-homologen x-Chromosom zusammenhängt; und (5) polygene oder multifaktorielle, die aus Mutationen in verschiedenen Genen resultieren oder aus der Wechselwirkung mehrerer Umweltfaktoren entstehen, daher das Highlight für die Amazonasregion Brasiliens, aufgrund ihrer großen Eigenart und Vielfalt ökologischer und nichtökologischer Umgebungen, die direkt mit dem Leben der Menschen verbunden sind und erheblich zu phänotischen Veränderungen in mehreren Genen beitragen können. , zum Beispiel koronare Herzerkrankungen, Krebs und Schizophrenie (LOBO, 2008; BERTOLLO et al., 2013; NUSSBAUM et al., 2016).

Genetische Erkrankungen können auch aus chromosomalen Veränderungen stammen, die als folgende Werte klassifiziert werden: (1) numerische Mutationen, bei denen sich die Anzahl der Chromosomen in der Zelle ändert; und (2) strukturelle Mutationen, bei denen sich Form oder Größe der Chromosomen ändert. Es gibt auch Fälle, in denen eine Mischung von Zelllinien auftreten kann, mit dem Vorhandensein einer normalen Linie und einem anderen veränderten, einem Ereignis namens Mosaikismus (BORGES-OSÓRIO; ROBINSON, 2013).

Die chromosomalen Variationen des numerischen Charakters werden in zwei Hauptkategorien eingeteilt: Euploidien und Aneuploidien. Euploidien beinhalten ganze Genome, die alle Chromosomen eines Individuums beeinflussen und eine Bedingung sind, die mit dem Leben unvereinbar ist. Aneuploide beinhalten

RC: 79877

Disponível em: https://www.nucleodoconhecimento.com.br/gesundheit/medizinische- 
Veränderungen der Chromosomenzahl, mit Erhöhung oder Verlust eines oder mehrerer Chromosomen, ohne die Haploid-Set (JORDE; CAREY; BAMSHAD, 2016).

Bei Männern sind diese chromosomalen Veränderungen, insbesondere bei größeren Chromosomen, fast immer tödlich, anders als bei kleineren Chromosomen, wie Chromosom 21, das in der Zelllinie bleiben kann, obwohl es zu Geburten mit körperlichen und geistigen Fehlbildungen führt. Unter den Folgen, die durch Chromosomenveränderungen verursacht werden, gibt es Down-, Edwards-, Patauund Klinefelter-Syndrome (ZANELLA; TERENCIO, 2016).

Aus dem Vorstehenden ist zu erwähnen, dass, da genetische Krankheiten eine ungeheilte Erkrankung sind, die Nachbeobachtung ein wesentlicher Bestandteil der Behandlung ist und das ganze Leben des Patienten dauert. Daher zielt die vorliegende Studie darauf ab, das Profil von Patienten zu bestimmen, die in einer GM-Ambulanz in einem Universitätszentrum von Belém, Para, Amazonien, beobachtet werden.

\section{MATERIAL UND METHODE}

Dies ist eine beschreibende und retrospektive Studie, in der kranken Aufzeichnungen von Patienten, die von Januar 2014 bis März 2020 behandelt wurden, in der GMAmbulanz des Zentrums für medizinische Spezialitäten eines Universitätszentrums in der Stadt Belém, Para, analysiert wurden. Alle Patienten (die ihre Anonymität aufrechterhielten) unterzeichneten ein Autorisierungsformular, damit ihre jeweiligen Krankenakten zur Datenerhebung für die Entwicklung dieser Forschung analysiert werden konnten. Dieses Projekt wurde von der Ethikkommission für Forschung mit Menschen eingereicht und genehmigt, gemäß der Genehmigungsstellungnahme der Nummer 2.917.287. Neben dem Protokoll dieser Studie folgten die Normen der Resolution Nr. 466/12 des Nationalen Gesundheitsrates Brasiliens (BRASIL, 2012), in der Standards für die Forschung am Menschen festgelegt werden.

RC: 79877

Disponível em: https://www.nucleodoconhecimento.com.br/gesundheit/medizinischegenetik 
Die Krankenakten von Patienten, die nur während des für diese Forschung genannten Zeitraums behandelt wurden, wurden aufgenommen, und die Krankenakten von Patienten, die in Ambulanzen anderer medizinischer Fachrichtungen gesehen wurden, wurden ausgeschlossen. Das Profil jedes Patienten wurde für die folgenden Variablen analysiert: Geschlecht, Alter, ethnische Zugehörigkeit, Herkunft, Herkunft der medizinischen Überweisung, Diagnose, Alter bei der Diagnose, Alter der Mutter bei der Geburt, Komplikationen in der Schwangerschaft, genetische Beratung und Nachsorge durch das multidisziplinäre Team.

Die Patienten wurden nach alterweise nach WHO-Kriterien bei Kindern (bis 9 Jahre), Jugendlichen (10 bis 19 Jahre), Erwachsenen (20 bis 59 Jahre) und älteren Menschen (ab 60 Jahren) klassifiziert.

Die Datenerhebung auf der Grundlage der Analyse von Krankenakten wurde anhand des eigenen Fragebogens der Forscher durchgeführt, der objektive und subjektive Fragen enthielt. Die Daten wurden dann tabellarisch erfasst und mit Hilfe des Microsoft Excel 2016-Programms analysiert.

\section{ERGEBNISSE}

In diesem Zeitraum wurden 101 Krankenakten analysiert. Davon waren 49 männliche und 51 weibliche Patienten (Abbildung 1). Darüber hinaus wurde festgestellt, dass die meisten Besuche für Kinder mit 41,5 \% der Gesamtzahl bestimmt waren, gefolgt von $31,68 \%$ bei Jugendlichen und 20,79 \% für Erwachsene. Den niedrigsten Prozentsatz stellten die älteren Menschen mit 5,94\% der Besuche dar (Abbildung 2). Es ist erwähnenswert, dass von der Gesamtzahl der Kinder die höchste Häufigkeit von Aufzeichnungen von Patienten im Alter von 4 Jahren war, die bei der Analyse von 10 Krankenakten gefunden wurden. In den anderen Altersgruppen war die Verteilung zwischen den Altersgruppen ähnlich.

RC: 79877

Disponível em: https://www.nucleodoconhecimento.com.br/gesundheit/medizinischegenetik 
Abbildung 1: Verteilung der Patienten, die in der medizinischen Genetik-Ambulanz nach Geschlecht beobachtet wurden.

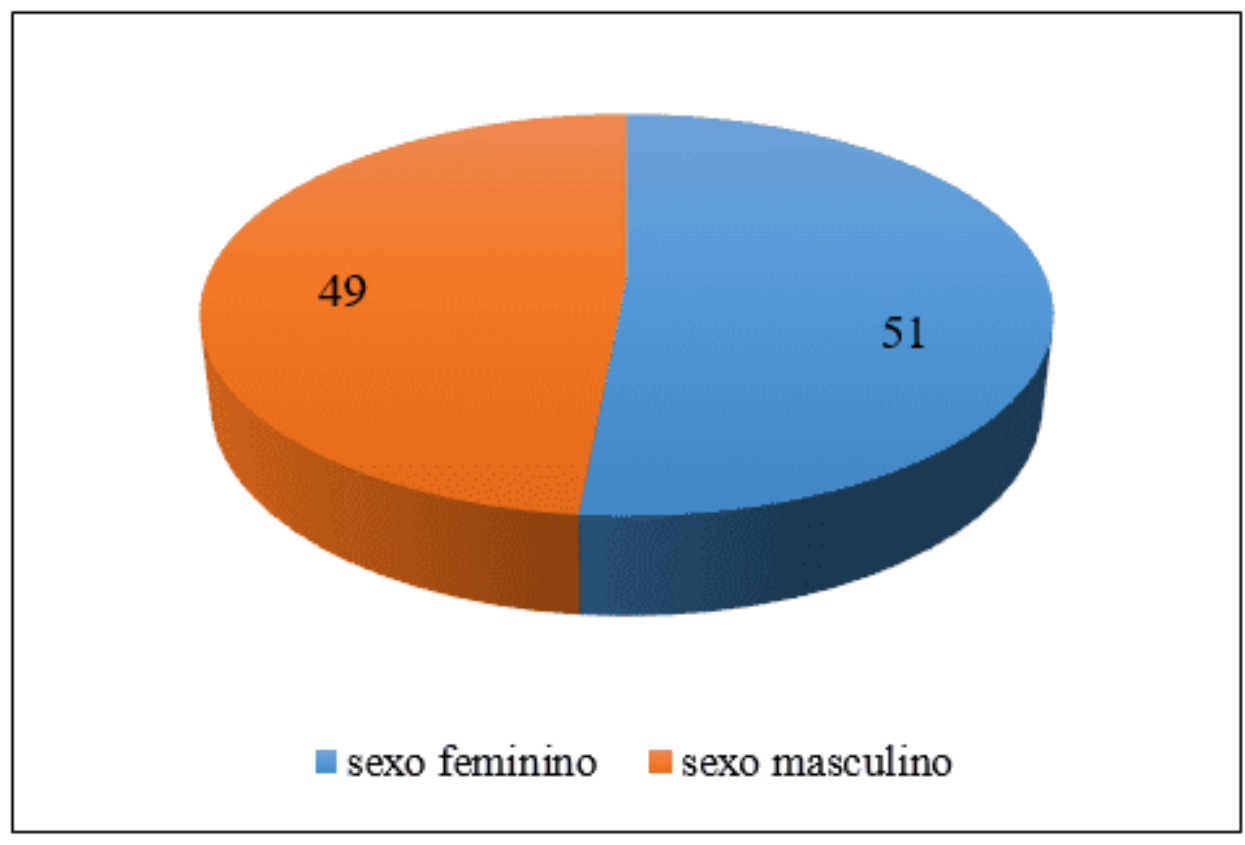

Quelle: Suchdaten.

Abbildung 2: Verteilung der Patienten, die in der medizinischen Genetik-Ambulanz beobachtet wurden, nach Altersgruppen.

RC: 79877

Disponível em: https://www.nucleodoconhecimento.com.br/gesundheit/medizinischegenetik 


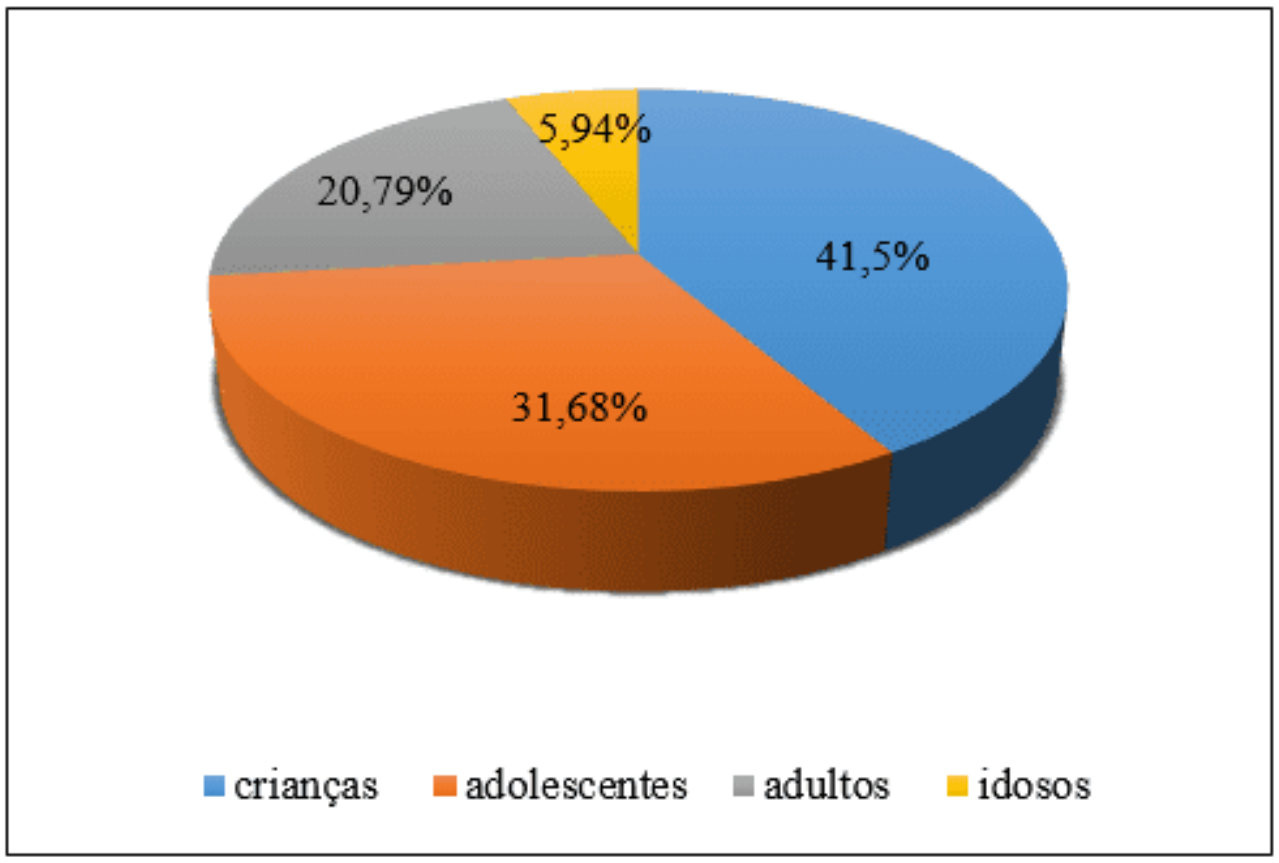

Quelle: Suchdaten.

Hinsichtlich der ethnischen Zugehörigkeit wurden bei der Analyse von Krankenakten die Variablen "weiß" und "braun" gefunden, ohne Aufzeichnungen über schwarze Patienten, abgesehen davon, dass sie Krankenakten hatten, ohne die Frage auszufüllen. So gab es 10 weiße Patienten, 78 braune Patienten und 13, in denen die Informationen nicht enthalten waren.

Belém war die am weitesten verbreitete Stadt in der Natürlichkeit der Patienten. Die Hauptstadt von Para konzentrierte sich auf 61 Datensätze, gefolgt von Abaetetuba mit 7 Patienten. Es wird betont, dass die Ambulanz in der analysierten Zeit besucht, vor allem Städte, die die Metropolregion Belém bilden. Nur ein Fall war eine Ausnahme: der eines Patienten, der vom Genetikdienst einer Universität in Campinas im Bundesstaat Sao Paulo verwiesen wurde. Weitere Städte der Studie waren Altamira (1), Ananindeua (4), Barcarena (3), Benevides (1), Bragana (3), Cameté (4), Capanema (1), Castanhal (2), Cotijuba (1 ), Marabé (1), Marituba (1),

RC: 79877

Disponível em: https://www.nucleodoconhecimento.com.br/gesundheit/medizinischegenetik 
Mosqueiro (2), Ourém (1), Paragominas (3), Portel (1), Santo Antonio do Taré (1), Tucurué (1) und Vigia (1) (Abbildung 3).

Abbildung 3: Verteilung der Patienten, die in der medizinischen Genetik-Ambulanz beobachtet wurden, nach Herkunft.

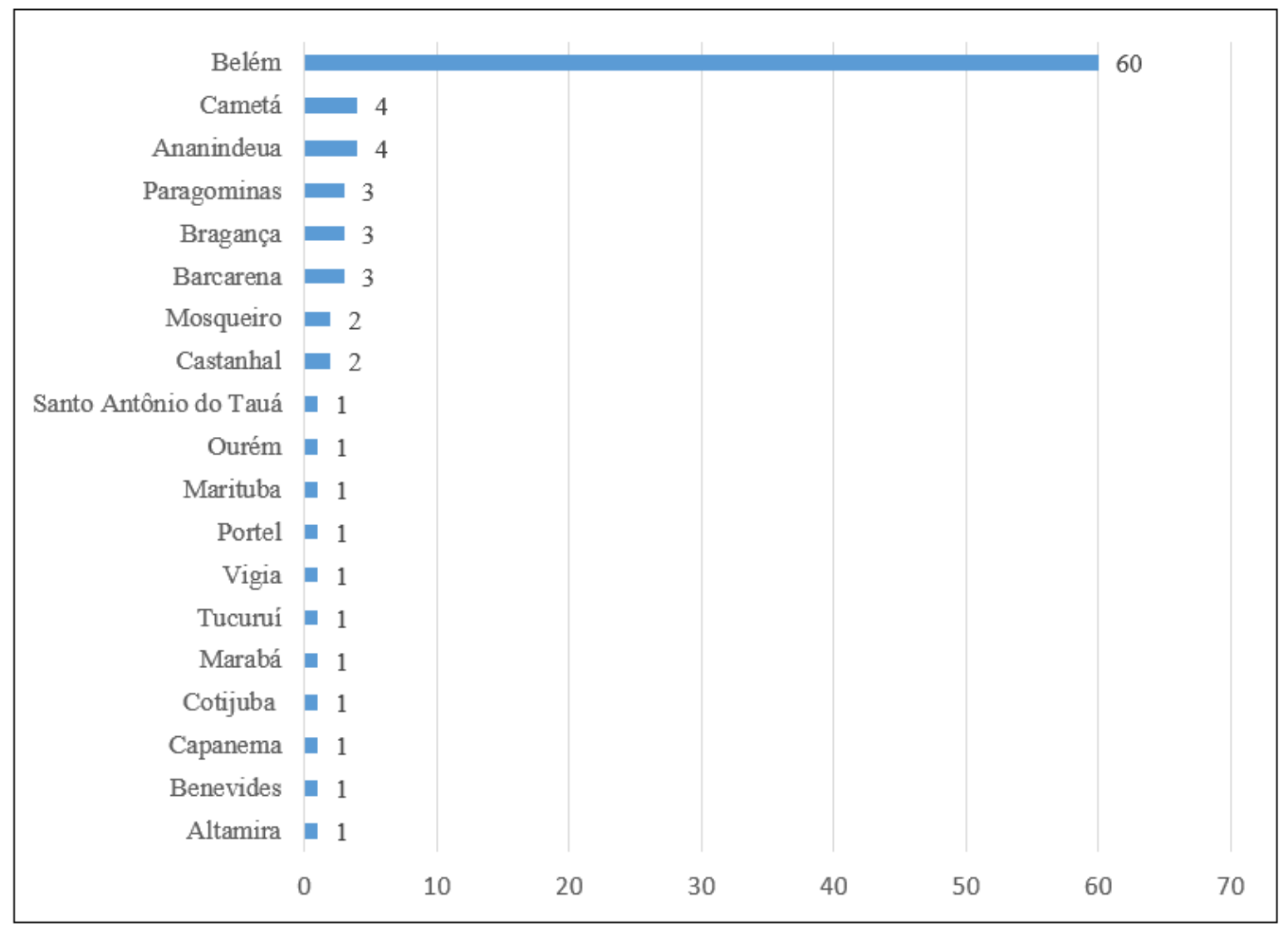

Quelle: Suchdaten.

Es wurde auch festgestellt, dass Endokrinologie und Neurologie sind die Spezialitäten mit der höchsten Anzahl von medizinischen Überweisungen an die GMAmbulanz. Die Neurologie verwies auf 26 Patienten mit einer Dominanz von Kindern, von denen 25 pädiatrische Patienten waren (10,89\%) und nur 1 Erwachsener. Die Endokrinologie schickte 22 Patienten. Es gibt jedoch auch eine große Anzahl von Krankenakten, in denen diese Informationen nicht gefunden wurden, insgesamt

RC: 79877

Disponível em: https://www.nucleodoconhecimento.com.br/gesundheit/medizinischegenetik 
$26,73 \%$. Darüber hinaus waren die anderen medizinischen Fachrichtungen, die sich auf GM bezogen, Die Gynäkologie (2), die Mastologie (2), die Neonatologie (2), die Klinische Medizin (1), die Dermatologie (1), die Gastropädiatrie (1), die Genetik der Stadt Campinas (1), die Orthopädie (1), die Pulmonologie (1), die Rheumatologie (1), die Gesundheit älterer Menschen (1) und die Grundgesundheitseinheit (1) (Abbildung $4)$.

Abbildung 4: Verteilung der Patienten, die in der medizinischen Genetik-Ambulanz durch Überweisung beobachtet wurden.

RC: 79877

Disponível em: https://www.nucleodoconhecimento.com.br/gesundheit/medizinischegenetik 


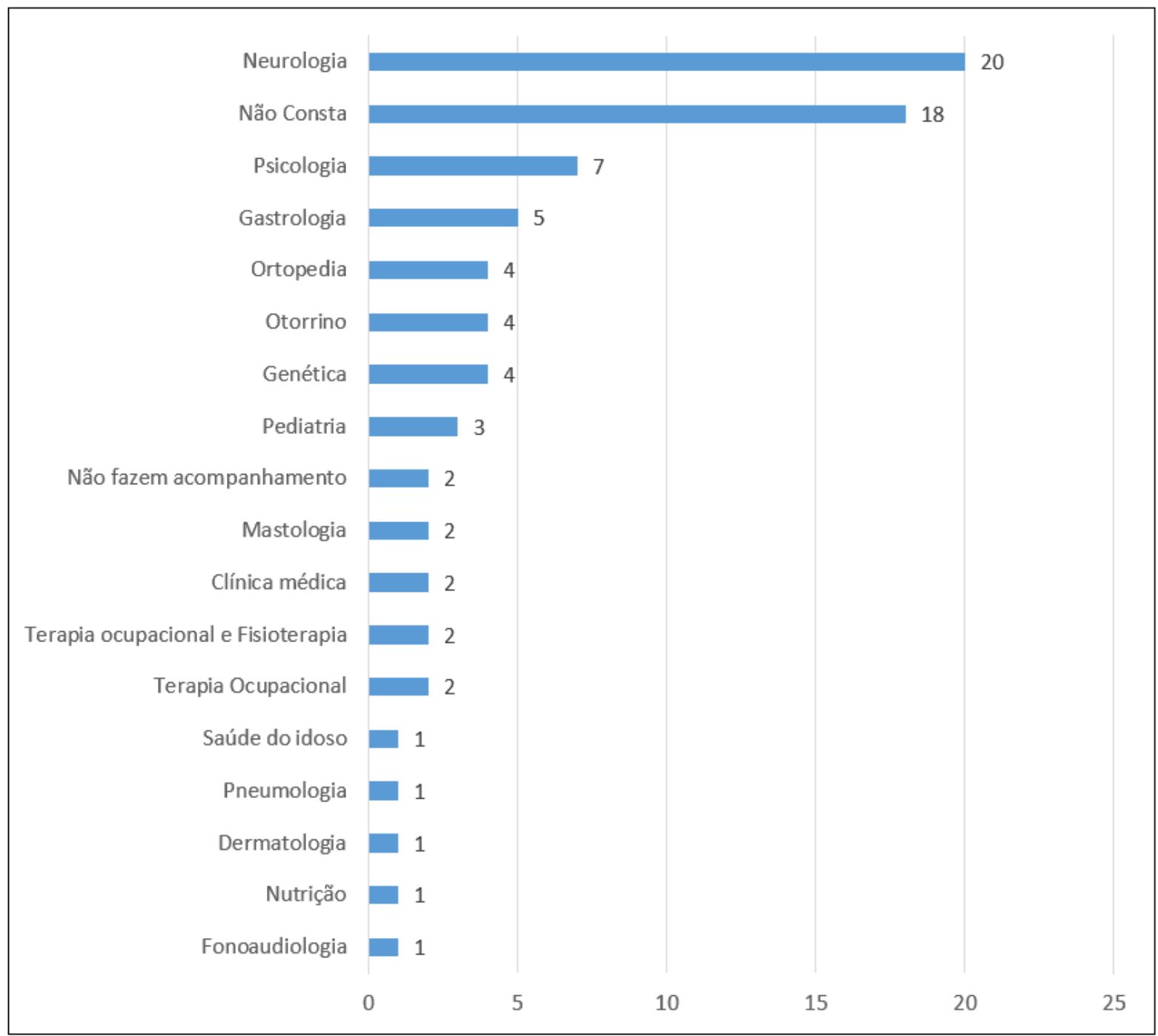

Quelle: Suchdaten.

Die Diagnosen waren diversifiziert. Sie wurden selten isoliert gesehen und wurden oft mit einer anderen Erkrankung in Verbindung gebracht. In der GM-Ambulanz gab es eine leichte Prävalenz der neuropsychomotorischen Entwicklungsverzögerung (ADNPM), mit 8 Datensätzen und in nur 2 Fällen war ADNPM die einzige Krankheit, die beim Patienten diagnostiziert wurde. In den anderen 6 wurde es mit kurzer Statur und Dysmorphie (3), angeborener Hypothyreose (1) und frühreifer Pubertät (2) in Verbindung gebracht. Einige Syndrome wurden gefunden, dysmorphes Syndrom (3), Noonan-Syndrom (3), Cornelia-Lange-Syndrom (2), Sotos-Syndrom (2), Charge-

RC: 79877

Disponível em: https://www.nucleodoconhecimento.com.br/gesundheit/medizinischegenetik 
Syndrom (1), Marfan-Syndrom (1), Aarskog-Syndrom (1), Down (1), BeckwithWiedermann-Syndrom (1), Turner-Syndrom (1), Prader-Willi-Syndrom (1), WilliamsSyndrom (1) und hyperkinetisches Syndrom im Zusammenhang mit Aufmerksamkeits-Hyperaktivität (1). Es wurden fünf Fälle von Chromosom s46, XY und weitere 5 Fälle von einer geistigen Behinderung festgestellt. Zusätzlich zu 4 Aufzeichnungen über die Untersuchung der Zyka-Virus-Embryopathie, 3 Untersuchungen der Familienkrankheit, von denen 2 Brustkrebs bei Schwestern. Angeborene Stoffwechselfehler stellten 4 Fälle dar, sowie eine kurze Statur der autosomal dominanten Vererbung. Acondroplasie, X-fragile und Epilepsie im Zusammenhang mit kognitivem Defizit betrug 3 Patienten pro. Auch mehrere endokrine Neoplasie Typ 1 und multiple endokrine Neoplasie Typ 2A, rechte einseitige Mikrotia, angeborene Megakolon mit Skelettveränderungen, Mahnart partielle Lipodystrodie mit Oligomenorrhoe, distale erbliche Neuropathie und Neurofibromatose präsentiert 1 Fall jeweils (Abbildung 5).

Andere Bedingungen wurden gefunden: Multiple Exostose (2), angeborene Nebennierenhyperplasie (2), Methylmalonsäureämie (1), X-verknüpfte Adrenoleukodystrophie (1), multifaktorielle Verhaltensänderung (1), distale Dominanz Arthrogripose im Zusammenhang mit Anzeichen bri da (1), Verzögerung der sexuellen Entwicklung aufgrund von Hypogonadismus (1), nicht-fibrotischer Bronchiektase (1), Dohi-Krankheit (1), Wachstumshormonmangel mit geringer Knochenmineraldichte mit myasthenischem Syndrom im Zusammenhang mit Kearnes-Sayre-Syndrom (1) , zentrale Hypothyreose mit Standseilbahn Myelose und epheskulärem Mikroadenom (1), medullärem Schilddrüsenkarzinom (1), nichtprogressiver chronischer Enzephalopathie (1), Mukoviszidose (1), Lymphödemassoziierter Genodermatose (1) und Hypodystrophie (1) (Abbildung 5). Innerhalb des Datenerhebungszeitraums wurden noch 10 Patienten untersucht oder ohne abgeschlossene Diagnose.

RC: 79877

Disponível em: https://www.nucleodoconhecimento.com.br/gesundheit/medizinische- 
Abbildung 5: Verteilung der Patienten, die in der medizinischen Genetik-Ambulanz beobachtet wurden, nach Diagnose.

RC: 79877

Disponível em: https://www.nucleodoconhecimento.com.br/gesundheit/medizinischegenetik 


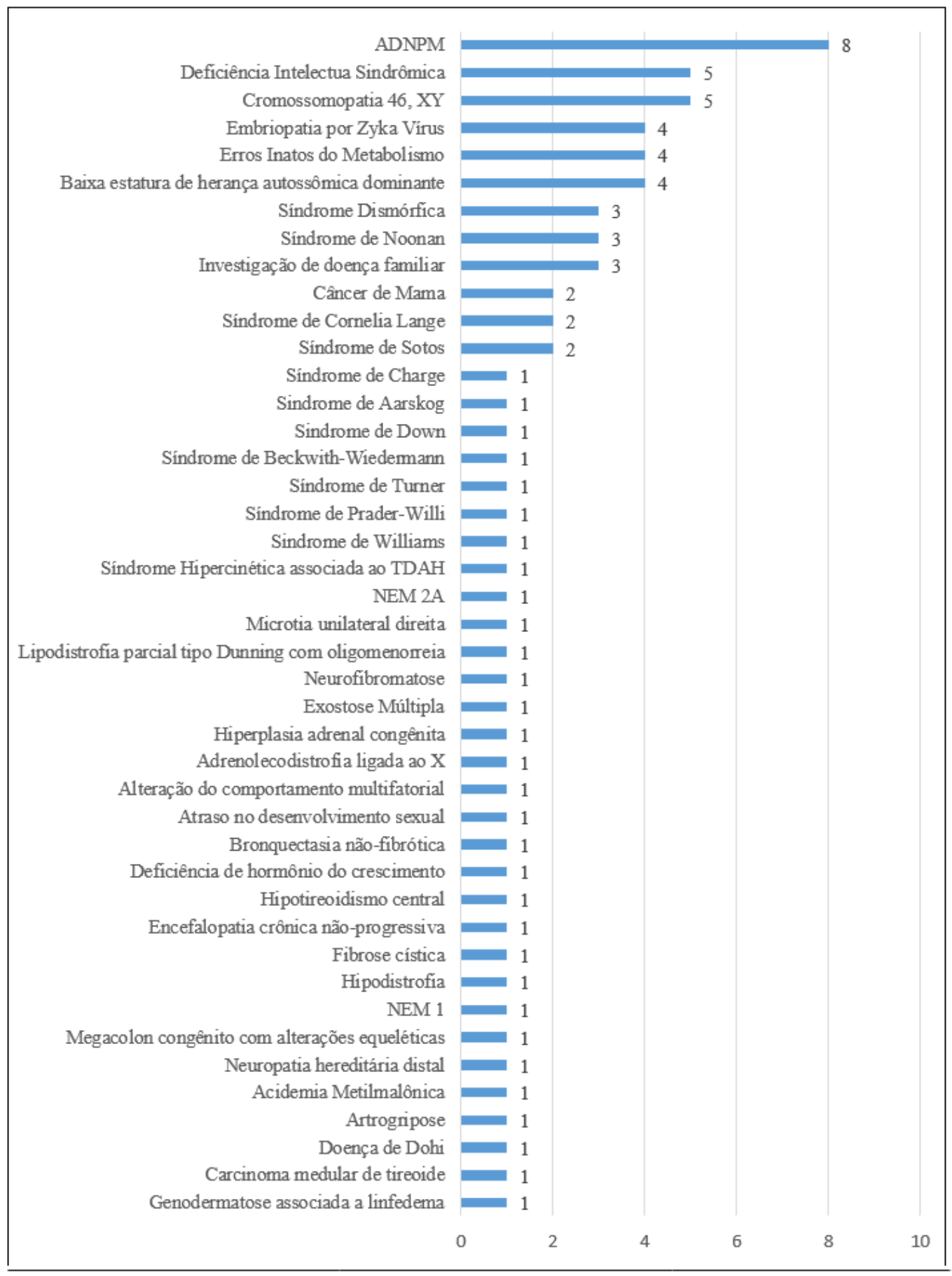

Quelle: Suchdaten.

In 42 Krankenakten war das Alter bei der Diagnose nicht vorhanden, 2 Patienten wurden noch im Mutterleib und 4 weitere bei der Geburt diagnostiziert. Weitere 11

RC: 79877

Disponível em: https://www.nucleodoconhecimento.com.br/gesundheit/medizinische- 
Patienten wurden unter 2 Jahren diagnostiziert; 13 Patienten im Alter von 2 bis 9 Jahren; 18 in der Pubertät, mit einer höheren Zahl im Alter von 14 Jahren, mit 6 Diagnosen. In der Erwachsenenaltersgruppe gab es 11 Diagnosen und nur 1 bei einem älteren Patienten im Alter von 63 Jahren (Abbildung 6).

Abbildung 6: Verteilung der Patienten, die in der medizinischen Genetik-Ambulanz beobachtet wurden, nach Alter bei der Diagnose.

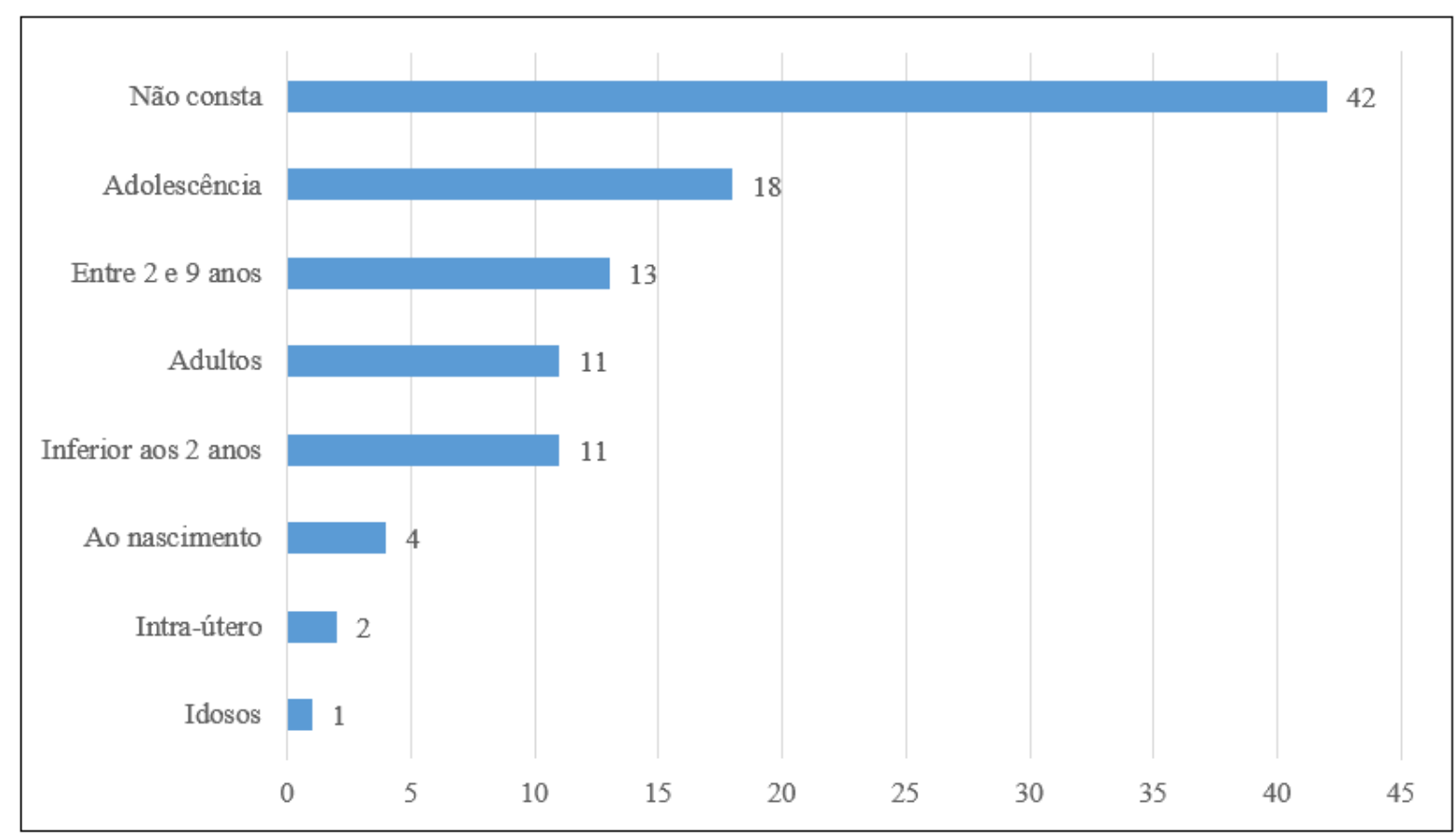

Quelle: Suchdaten.

Von den 101 Patienten hatten nur 16 genetische Beratung und in den übrigen 85 gab es keine Aufzeichnungen über diese Informationen.

Am häufigsten folgten die Patienten die Endokrinologie, 21 Aufzeichnungen, gefolgt von der Neurologie mit 20 Patienten und der Psychologie folgten 7 Patienten. Darüber hinaus wurden 5 Patienten mit Gastronomie, 4 mit Genetik, Otorrino und Orthopädie, weitere 3 mit Pädiatrie, 2 mit Ergotherapie, 2 für Physiotherapie und Ergotherapie gleichzeitig, 2 mit der Medizinischen Klinik, 2 mit Mastologie, 1 mit

RC: 79877

Disponível em: https://www.nucleodoconhecimento.com.br/gesundheit/medizinischegenetik 
Sprachtherapie, 1 mit Ernährung, 1 mit Dermatologie, 1 mit Pulmonologie und 1 mit Seniorengesundheit überwacht. Außerdem wurden in 18 Krankenakten die Variable nicht ausgefüllt, und 2 wurden nicht weiterverfolgt (Abbildung 7).

Schaubild 7: Verteilung der Patienten, die in der medizinischen Genetik-Ambulanz beobachtet wurden, nach Folgen.

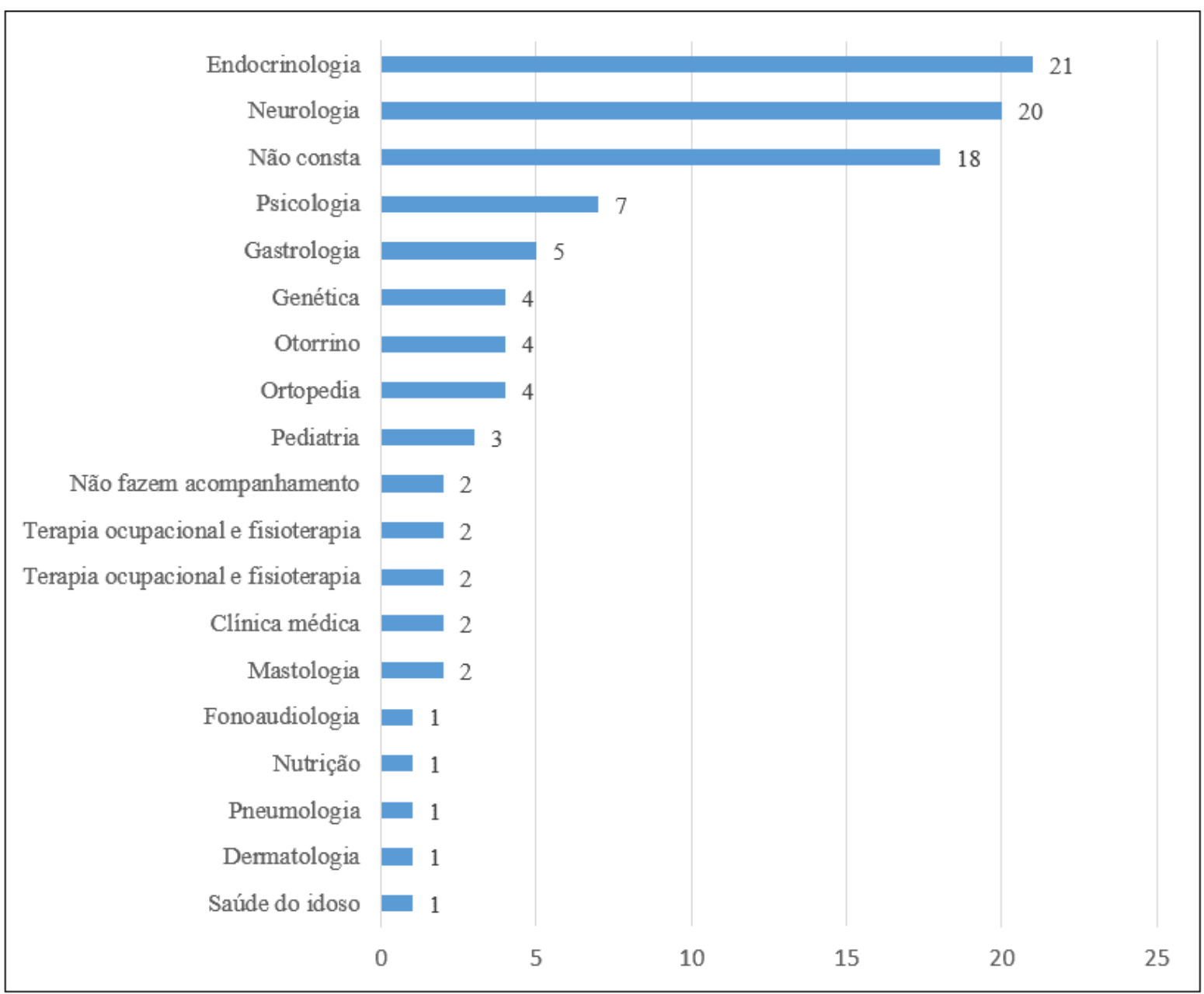

Quelle: Suchdaten.

Die Angaben über das Alter der Mutter fehlten in den meisten Krankenakten, nicht in $61,38 \%$ der Gesamtzahl. In den Krankenakten, in denen die Informationen vorhanden waren (38,62\%), betrug das alter mütterliche Alter zwischen 16 und 19

RC: 79877

Disponível em: https://www.nucleodoconhecimento.com.br/gesundheit/medizinischegenetik 
Jahren 7,92 \%, während die Altersgruppen zwischen 20 und 59 Jahren 30,7 \% der Aufzeichnungen ausmachten.

In 36 Krankenakten gab es keine Informationen über Komplikationen während der Schwangerschaft und in 25 Fällen gab es keine Komplikationen. Diejenigen, die hatten (40) waren vielfältig: Harnwegsinfektion (8), Gebärmutterblutung (6), Frühgeburt (5), Präeklampsie (4), Verzögerung der Kontraktionen der Vertreibung des Fötus (2), Oligohydramnio (2), perinatales Zyto Xia (1), zentrale Zyose (1), nephrotische Koliken (1), im Geburtskanal eingeschlossenes Kind (1), Plazentaablösung (1), Schmerzen während der Schwangerschaft (1), Alkoholkonsum und Rauchen (1), Krankenhausaufenthalt bei asthmamütterlicher Krise (1) , Hundebiss (1), Rauchen, Metrorragie und Kind mit Onfocele in USG - bei demselben Patienten - (1), Verwendung von Kapillarfarbstoff und Verfärbung von Körperhaaren (1), Windpocken (1) und Zyka-Müttervirus (1).

\section{DISKUSSION}

Belém ist eine brasilianische Gemeinde, Hauptstadt des Bundesstaates Para, in der nördlichen Region des Landes, genauer gesagt in der Region des brasilianischen Amazonas. Sie ist mit 1.492.745 EINWOHNER (INSTITUTO BRASILEIRO DE GEOGRAFIA E ESTATÍSTICA, 2020). Die Stadt verfügt über ein Wirtschafts- und Dienstleistungsnetz - einschließlich des Gesundheitswesens -, das den Städten ihrer Metropolregion dient.

Es wird davon ausgegangen, dass ein Genetiker-Arzt pro 100.000 Einwohner notwendig ist, einschließlich ambulanter, krankenhausärztlicher und genetischer Beratung (CARVALHO et al., 2016; CONSELHO FEDERAL DE MEDICINA, 2020). Nach Angaben des Bundesrates für Medizin (CONSELHO FEDERAL DE MEDICINA, 2020) praktizieren derzeit nur 3 Ärzte die Spezialität GM. Darüber hinaus arbeiten alle in der Hauptstadt von Para. So wird die Knappheit des Dienstes festgestellt, da die Spezialität der gesamten Kollektivität zugute kommt und das Gesundheitssystem

RC: 79877

Disponível em: https://www.nucleodoconhecimento.com.br/gesundheit/medizinische- 
beschleunigt. Denn die frühzeitige Diagnose genetischer Erkrankungen ermöglicht ein besseres Management der Patienten und verhindert oder minimiert mögliche Folgen und senkt auch die Kosten bei möglichen komplexeren Behandlungen.

Eine weitere relevante Daten, auf die in der Studie hingewiesen wird, ist der Mangel an genetischer Beratung durch das multidisziplinäre Team, das den Patienten unterstützt, da nur etwa 16\% der Patienten Zugang zu diesem Service hatten, für die anderen fehlten die Informationen. Diese Tatsache kann mit der Knappheit des Dienstes zusammenhängen, da nicht alle Patienten mit genetischen Erkrankungen über den Dienst verfügen, und es ist notwendig, das Versorgungsnetz für diese Öffentlichkeit zu erweitern, angesichts der Bedeutung einer multiprofessionellen Beratung, insbesondere im Hinblick auf die Prävention von Genotypen, die für Krankheiten oder Geburtsfehler verantwortlich sind (JORDE; CAREY; BAMSHAD, 2016).

Die Studie zeigte auch die hohe Rate der Überweisungen zwischen GM und anderen Spezialitäten des Medical Center, in dem die Forschung durchgeführt wurde. Die Spezialgebiete mit der höchsten Anzahl von Überweisungen waren Endokrinologie und Neurologie, neben der Überweisung von GM an andere Fachleute, wie Psychologen und Ergotherapeuten. Als berüchtigt die Bedeutung des multiprofessionellen Ansatzes der Patienten, da einige genetische Krankheiten mit einem oder mehreren Systemen des Organismus zusammenhängen (BERTOLLO et al., 2013).

In Bezug auf das mütterliche Alter bei der Geburt des Patienten enthielt mehr als die Hälfte der Krankenakten nicht die Informationen (62 Krankenakten), aber die Krankenakten, die diese Daten präsentierten, ergaben, dass das Alter der Mutter in 6 Krankenakten größer als 35 Jahre war. Diese Daten werden relevant, da Studien auf einen Zusammenhang zwischen fortgeschrittenem mütterlichem Alter und der Entwicklung genetischer Störungen hindeuten. Studien zeigen, dass die Prävalenz genetischer Komplikationen bei Föten von Müttern mit 20 Jahren 1 zu 1.000 beträgt,

RC: 79877

Disponível em: https://www.nucleodoconhecimento.com.br/gesundheit/medizinische- 
während dieser Anteil mit 35 Jahren 1 von 350 Schwangeren erreicht. Erneut die Bedeutung der genetischen Beratung untermauern (MENDES, 2017; BARBOZA et al., 2020).

\section{SCHLUSSFOLGERUNG}

Die Erstellung eines Profils für den Patienten, der in einer Medizinischen GenetikAmbulanz besucht wird, ist äußerst wichtig, da es aus diesem Wissen möglich wird, mögliche Fehler im Geleisteten zu identifizieren, zusätzlich zur Anpassung der ArztPatienten-Beziehung. Es wird auch betont, dass die korrekte Annahme und Durchführung der Behandlung und Nachbereitung einer genetischen Krankheit von persönlichen und sozialen Faktoren sowie von der Phase abhängt, in der die Krankheit die Kontinuität der Behandlung in Betracht zieht. Somit wird die Notwendigkeit bestätigt, das Profil des behandelten Patienten zu bestimmen.

So zeigten die Ergebnisse der vorliegenden Studie, wie wichtig es ist, die Krankenakten des Patienten, der in der Medizinischen Genetik-Ambulanz anwesend ist, angemessen zu füllen, um den Verlust wichtiger Informationen zu vermeiden, die helfen können, die Diagnose zu bestimmen oder bei der Durchführung des Falles bei der Nachbeobachtung des Patienten zu helfen.

Ein weiterer wichtiger Faktor, der während der Studie hervorgehoben wird, ist die häufige Überlappung von Diagnosen, da Patienten in der Regel mehr als eine Pathologie besuchen, ist es wichtig, die Pflege zu spezialisieren, wobei die Aufmerksamkeit auf diese Tatsache zu lenken, um mögliche iatrogene und interkorréncias zu vermeiden, mit Wechselwirkungen mit Medikamenten während der Behandlung.

\section{VERWEISE}

RC: 79877

Disponível em: https://www.nucleodoconhecimento.com.br/gesundheit/medizinischegenetik 
BARBOZA, B. P.; CALIL, C.; TRIGO, I. G. P. F.; ELLER, J. X.; SILVA, L. R.; VAZ, M. R.; ESTEVES, A. P. V. S. Idade materna avançada e seus desfechos. Cadernos da Medicina-UNIFESO, v. 2, n. 3, 2020.

BeRTOLlo, E. M. G.; CASTRO, R; CINTRA, M. T. R.; PAVARINO, É. C. O processo de aconselhamento genético. Arq. Cienc. Saúde, v. 20, n. 1, p. 30-6, 2013.

BORGES-OSÓRIO, M. R; ROBINSON, W. M. Genética Humana. 3ae edição, Porto. Alegre, Editora Artmed, 2013.

CARVALHO, A. C.; VIEIRA, E.; ALTENHOFEN, T. M. G.; JUNG, M. S. Perfil clínicoepidemiológico dos pacientes atendidos pelo Serviço de Genética Médica do Ambulatório Materno Infantil da Universidade do Sul de Santa Catarina. Arquivos Catarinenses de Medicina, v. 45, n. 2, p. 11-24, 2016.

CONSELHO FEDERAL DE MEDICINA. Disponível em: < https://sistemas.cfm.org.br/normas/arquivos/pareceres/BR/2018/17_2018.pdf > Acesso em 20 de março de 2020.

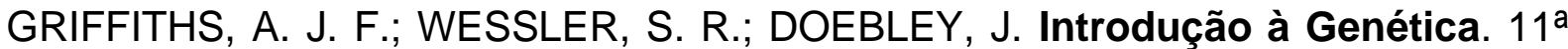
edição. Guanabara Saúde Didático, 2016.

INSTITUTO BRASILEIRO DE GEOGRAFIA E ESTATÍSTICA. Disponível em: $<$ https://www.ibge.gov.br/cidades-e-estados/pa/belem.html> Acesso em: 20 de março de 2020.

JORDE, L. B.; CAREY, J. C.; BAMSHAD, M. J.; Genética Médica. 5ª edição, Rio de Janeiro, Editora Elsevier. 2016.

LOBO, I. Environmental influencies on gene expression. Nature Education 1(1):39, 2008.

RC: 79877

Disponível em: https://www.nucleodoconhecimento.com.br/gesundheit/medizinischegenetik 
MENDES, C. C. Estudo genético e epigenético de fatores de risco materno para a síndrome de Down. 2017. 32 f. Tese (Programa de Pós-Graduação em Ciências da Saúde) - Faculdade de Medicina de São José do Rio Preto, São José do Rio Preto.

NUSSBAUM, R. L.; MCINNES, R. R.; HUNTINGTON, F. W.; THOMPSON, M. W. Thompson \& Thompson. Genetics in Medicine. Philadelphia: Elsevier. 2016.

SOCIEDADE BRASILEIRA DE GENÉTICA MÉDICA. Disponível em: $<$ http://www.sbgm.org.br>. Acesso em: 20 março, 2020.

ZANELLA, J.; TERENCIO, M. L. Genética clínica: investigação dos padrões de herdabilidade de doenças humanas-mutações genéticas e cromossômicas. Artigo completo publicado em: Anais do V Encontro de Iniciação Científica e I Encontro Anual de Iniciação ao Desenvolvimento Tecnológico e Inovação - EICTI. 2016.

Eingereicht: März, 2021.

Genehmigt: März 2021.

RC: 79877

Disponível em: https://www.nucleodoconhecimento.com.br/gesundheit/medizinischegenetik 